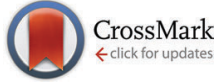

Cite this: Phys. Chem. Chem. Phys., $2016,18,16527$

Received 11th May 2016, Accepted 1st June 2016 DOI: $10.1039 / c 6 c p 03203 f$

www.rsc.org/pccp

\title{
High-pressure phase transitions in rubidium and caesium hydroxides $\dagger$
}

\begin{abstract}
Andreas Hermann*
A computational investigation of the high-pressure phase sequence of the heaviest alkali hydroxides, $\mathrm{RbOH}$ and $\mathrm{CsOH}$, shows that the phase diagram of both compounds is richer than hitherto thought. First-principles calculations suggest, based on energetics and comparisons to experimental diffraction and spectroscopy signatures, that the high-pressure phase $\mathrm{RbOH}-\mathrm{VI}$, stable above $6 \mathrm{GPa}$ in experiment, should be assigned the $\mathrm{KOH}-\mathrm{VI}$ structure type, and features localised hydrogen-bonded $(\mathrm{OH})_{4}$ units. Meanwhile, a new high-pressure phase $\mathrm{CsOH}-\mathrm{VII}$ is predicted to be stable above $10 \mathrm{GPa}$ in an isosymmetric phase transition that, like $\mathrm{RbOH}-\mathrm{Vl}$, marks the transition from layered to three-dimensional network structures under increased compression. Both new phases highlight an unexpected flexibility of hydrogen bond network formation in a series of compounds that seemingly only vary in the cation size, and potential consequences for similar systems, such as water-carrying minerals, are discussed briefly.
\end{abstract}

\section{Introduction}

Alkali hydroxides, chemical composition $\mathrm{MOH}$ with $\mathrm{M}$ an alkali metal from lithium to caesium, are intriguing compounds at the interface of ionic and hydrogen bonding. Their crystal structures at high temperatures are driven by ionic interactions between $\mathrm{M}^{+}$and $\mathrm{OH}^{-}$: just below the melting point, all alkali hydroxides - bar lithium hydroxide, $\mathrm{LiOH}$ - form a cubic "rotator" phase in the sodium chloride structure with freely rotating $\mathrm{OH}^{-}$ groups on one lattice site, and alkali cations on the other. ${ }^{1-5}$ Around room temperature, hydrogen bonds between the hydroxyl groups are stabilised, which break the cubic symmetry and result in monoclinic, orthorhombic, or tetragonal phases. ${ }^{6,7}$ The respective structures are - again, except for $\mathrm{LiOH}$ - protondisordered, and low-temperature proton-ordered modifications have been found in almost all protonated and deuterated compounds (except for $\mathrm{NaOH}){ }^{8-11}$ Despite these commonalities, microscopic details of the respective compounds' structures differ. For instance, the low-temperature modification of $\mathrm{RbOH} / \mathrm{D}$ has one-dimensional $\mathrm{OH} \cdots \mathrm{OH}$ chains in overall ferroelectric order, while $\mathrm{KOH} / \mathrm{D}$ and $\mathrm{CsOH} / \mathrm{D}$ show antiferroelectric order. ${ }^{12}$ Fig. $\mathrm{S} 1$ in the ESI $\dagger$ provides a schematic overview over this phase sequence.

It has been argued that moderate compression at room temperature could induce transitions to the low-temperature

Centre for Science at Extreme Conditions and SUPA, School of Physics and Astronomy, The University of Edinburgh, Peter Guthrie Tait Road, Edinburgh, EH9 3FD, UK. E-mail: a.hermann@ed.ac.uk

$\dagger$ Electronic supplementary information (ESI) available. See DOI: 10.1039/c6cp03203f modifications in these compounds, and such transitions (below $P=1 \mathrm{GPa}$ ) have indeed been found in all alkali hydroxides and deuteroxides. ${ }^{13-16}$ The compounds' responses to increased density (especially of the hydrogen bond network) are of particular interest. In a sense, alkali hydroxides represent the simplest models of hydrous minerals. Those are complex phases within the $\mathrm{MgO}-\mathrm{H}_{2} \mathrm{O}-\mathrm{SiO}_{2}$ and $\mathrm{Al}_{2} \mathrm{O}_{3}-\mathrm{H}_{2} \mathrm{O}-\mathrm{SiO}_{2}$ phase diagrams, and are important carriers and storage materials for water in Earth's mantle. ${ }^{17-19}$ Water in these minerals is mainly present in hydroxyl form, and can drastically alter the minerals' physical properties. The ladder of interactions that drive the formation and properties of hydrous minerals (ionic, covalent, and hydrogen bonding) is present in the alkali hydroxides as well, and studies of the behaviour of the latter under pressure can give us valuable insight into the behaviour of compressed hydrogenbonded networks.

Experimental high-pressure studies of the alkali hydroxides have been performed up to $P=15 \mathrm{GPa}$ in some of these compounds, close to mantle transition zone conditions. Phase transitions beyond the presumed low-temperature modifications, resulting in denser non-layered phases, have been reported: $\mathrm{NaOH}-\mathrm{V}$, stable above $0.8 \mathrm{GPa}$, with bifurcated hydrogen bonds; ${ }^{16} \mathrm{KOH}-\mathrm{VI}$, stable above $6.5 \mathrm{GPa}$, and RbOH-VI, stable above $6.3 \mathrm{GPa}$, which were both described as taking up a "anti$\mathrm{NaOH}$ " structure, though it was not made clear what that structure is. ${ }^{15}$ Recently, first-principles calculations together with neutron powder diffraction were able to identify the $\mathrm{KOH}-\mathrm{VI}$ structure as having an unusual hydrogen-bond network topology, with localised $(\mathrm{OH})_{4}$ units in a matrix of potassium cations. ${ }^{20}$ Likewise, the high-pressure phase LiOH-III, stable above $0.7 \mathrm{GPa}$, 
was shown in calculations (analysing neutron diffraction patterns, vibrational and energetic properties) to be a new structure type with linear $\mathrm{OH} \cdots \mathrm{OH}$ chains. Calculations also predicted a high-pressure phase LiOH-IV (above $17 \mathrm{GPa}$ ) that is structurally identical to $\mathrm{NaOH}-\mathrm{V}$. $^{21}$

Here, the thread of theoretical studies of the compressed alkali hydroxides is continued, and the high-pressure phase transitions exhibited by $\mathrm{RbOH}$ and $\mathrm{CsOH}$ are examined. It is suggested, on grounds of energetics as well as comparisons to energy-dispersive X-ray (EDX) and spectroscopic data, that $\mathrm{RbOH}-\mathrm{VI}$ has the same structure as $\mathrm{KOH}-\mathrm{VI}$, thus also featuring localised $(\mathrm{OH})_{4}$ units; and that $\mathrm{CsOH}$ undergoes an isosymmetric phase transition to a high-pressure phase at $10 \mathrm{GPa}$, which has not been detected in previous experiments.

\section{Computational methodology}

To search for potential high-pressure structures, ground-state crystal structure searches were performed for both $\mathrm{RbOH}$ and $\mathrm{CsOH}$, with four formula units per unit cell and at 10 and $20 \mathrm{GPa}$, respectively. The former is significantly above the onset of $\mathrm{RbOH}-\mathrm{VI}$, reported at $6.3 \mathrm{GPa}$, and the search unit cells correspond to the unit cell size deduced from the EDX data. They would also be able to describe any structure with one or two formula units per cell. Over 1200 structures were optimized in each structure search.

The evolutionary algorithm approach as implemented in the XTALOPT package was used in conjunction with scalar relativistic density functional total energy calculations using the Vasp package. ${ }^{22,23}$ This combined computational approach has proven very fruitful in recent years in computational studies of high-pressure systems, including hydrogen-rich and hydrogenbonded compounds. ${ }^{24-28}$ Candidate structures from the crystal structure searches, as well as other structures known in the alkali hydroxides, were optimized across the pressure range $P=1 \mathrm{~atm}$ to $20 \mathrm{GPa}$. Density functional calculations employed the PBE exchange-correlation functional to describe the electron-electron interaction and projector augmented wave (PAW) data sets to model the electron-ion interaction, ${ }^{29,30}$ using $\mathrm{Rb}\left(4 \mathrm{~s}^{2} 4 \mathrm{p}^{6} 5 \mathrm{~s}^{1}\right) \mathrm{Cs}\left(5 \mathrm{~s}^{2} 5 \mathrm{p}^{6} 6 \mathrm{~s}^{1}\right) \mathrm{O}\left(2 \mathrm{~s}^{2} 2 \mathrm{p}^{4}\right)$ and $\mathrm{H}\left(1 \mathrm{~s}^{1}\right)$ valence spaces, with cutoff radii of $2.5,2.5,1.52$, and $1.1 a_{\mathrm{B}}$, respectively. Spin-polarisation and spin-orbit effects were not included, as all systems studied here are effectively closed-shell, wide-gap insulators. A plane wave basis cutoff of $600 \mathrm{eV}$ and regular $k$-grids of density $20 / \AA^{-1}$ to sample reciprocal space were used to achieve convergence of total energies, and geometries were optimised until remaining force components were below $1 \mathrm{meV} \AA^{-1}$. Phonon calculations used the finite displacement method in conjunction with the supercell approach as implemented in the PHON code to obtain phonon densities of states, ${ }^{31}$ utilising supercells with up to 144 atoms and symmetryadapted central displacements of $\pm 0.02 \AA$. Density functional perturbation theory as implemented in the Quantum-ESPRESSO package was used to ascertain IR and Raman activity of zonecentered modes. $^{32}$

\section{Results and discussion}

\section{1 $\mathrm{RbOH}$ and $\mathrm{CsOH}$ at atmospheric pressure}

The low-temperature phases of $\mathrm{RbOH}$ and $\mathrm{CsOH}$, denoted $\mathrm{RbOH}$ $\mathrm{IVb}$ and $\mathrm{CsOH}-\mathrm{IVc}$ respectively, are very similar: in one interpretation, they feature kinked one-dimensional $\mathrm{OH} \cdot \cdots \mathrm{OH}$ chains running through channels of a cation matrix, see Fig. 1. Orthorhombic RbOH-IVb, space group $C m c 2_{1}$, has ferroelectrically ordered chains, while CsOH-IVc, space group $P 2_{1} 2_{1} 2_{1}$, has antiferroelectrically ordered chains (in Fig. 1(b), these point along the $c$ axis, alternately into and out of the paper plane). A very common alternative view of both structures is that they are layered compounds, as schematically shown in Fig. 1 (c): (Rb,Cs) $\mathrm{O}_{5}$ polyhedra form layers of edge-sharing square planar pyramids, with their bases connected to adjacent layers through hydrogen bonds. The topology shown in Fig. 1(c) is the same in RbOH-IVb and $\mathrm{CsOH}-\mathrm{IVc}$, the two phases only differ in the relative orientations of the inter-layer hydrogen bonds.

In the present calculations, the structural parameters of both $\mathrm{RbOH}-\mathrm{IVb}$ and $\mathrm{CsOH}-\mathrm{IVc}$, as determined by neutron powder diffraction at atmospheric pressure are reproduced very well, see Table 1 . Lattice constants are mostly in agreement by $0.1 \AA$, and atomic positions by 0.02 in relative coordinates.

\subsection{High-pressure phase evolution in $\mathbf{R b O H}$}

In Fig. 2, the ground state enthalpies of formation (relative to $\mathrm{RbOH}-\mathrm{IVb}$ ) of various $\mathrm{RbOH}$ phases are plotted as function of pressure. The figure includes known alkali hydroxide phases (found at atmospheric or higher pressure) as well as results from crystal structure searches at $P=10 \mathrm{GPa}$. Naturally, the atomistic models here are restricted to proton-ordered phases.

The calculations correctly reproduce $\mathrm{RbOH}-\mathrm{IVb}$ as the ground state of $\mathrm{RbOH}$ at low pressures. Only slightly higher in enthalpy is the CsOH-IVc structure type that, as discussed above, only differs in the anti-ferroelectric order of the $\mathrm{OH}$ chains, but is otherwise very similar. At $P=10 \mathrm{GPa}$, two structures very close in enthalpy are found to become more stable than RbOH-IVb. Both are tetragonal, of $I 4 / \mathrm{m}$ and $P 4 / m n c$ symmetry, and emerged from the crystal structure searches. These are the same structures that were found as high-pressure candidates in potassium hydroxide
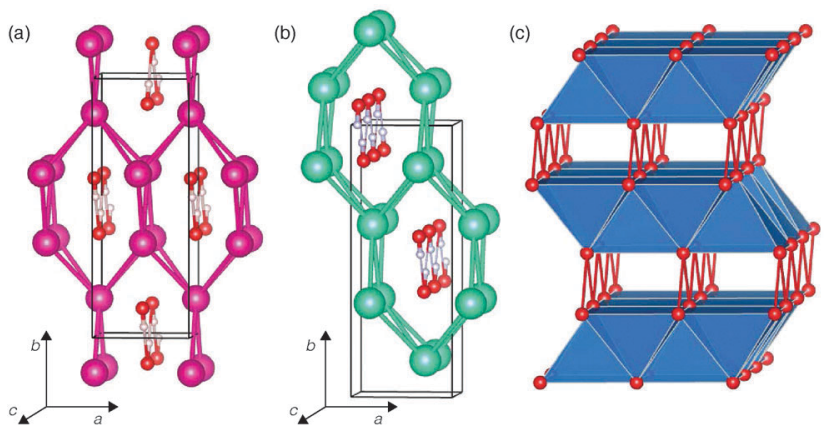

Fig. 1 Low-temperature structures of (a) $\mathrm{RbOH}-\mathrm{IVb}$, (b) $\mathrm{CsOH}-\mathrm{IVc}$, and (c) their common bilayered structure. Purple (green, red, white) spheres denote $\mathrm{Rb}(\mathrm{Cs}, \mathrm{O}, \mathrm{H})$ atoms, respectively. Protons are omitted in (c), and only shortest interlayer $\mathrm{O}-\mathrm{O}$ separations are drawn as connected. 
Table 1 Crystal structures of low-temperature $\mathrm{RbOH}$ and $\mathrm{CsOH}(\mathrm{D})$, comparing this work with neutron diffraction data from ref. 4 and 5

\begin{tabular}{|c|c|c|}
\hline Phase & Lattice constants $[\AA]$ & Atomic positions \\
\hline $\begin{array}{l}\text { RbOH, this } \\
\text { work }\end{array}$ & $a=4.221, b=11.100, c=4.251$ & $\begin{array}{l}\mathrm{Rb}(0,0.139,0.257) \\
\mathrm{O}(0,0.398,0.289) \\
\mathrm{H}(0,0.464,0.135)\end{array}$ \\
\hline RbOD, $15 \mathrm{~K}$ & $a=4.1114, b=10.991, c=4.1965$ & $\begin{array}{l}\mathrm{Rb}(0,0.138,0.25) \\
\mathrm{O}(0,0.389,0.282) \\
\mathrm{D}(0,0.463,0.149)\end{array}$ \\
\hline
\end{tabular}

$\mathrm{CsOH}$, this $\quad a=4.446, b=11.671, c=4.548$

work

$\operatorname{Cs}(0,0.139,0.252)$

$\mathrm{O}(0,0.402,0.292)$

$\mathrm{H}(0,0.462,0.141)$

CsOD, $23 \mathrm{~K} \quad a=4.312, b=11.514, c=4.462$

Cs $(0.01,0.14,0.25)$

$\mathrm{O}(0,0.393,0.269)$

$\mathrm{D}(0,0.462,0.143)$

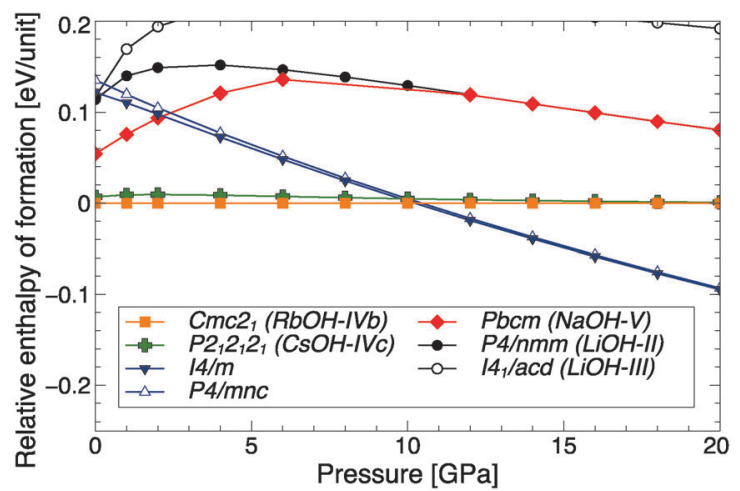

Fig. 2 Relative ground state enthalpies of formation per $\mathrm{RbOH}$ formula unit, as function of pressure.

and that formed the basis of a successful refinement of neutron powder diffraction data of $\mathrm{KOH}-\mathrm{VI} .^{20}$ The $\mathrm{NaOH}-\mathrm{V}$ phase, suggested in EDX experiments, is not energetically competitive at high pressures, and neither is any of the other known alkali hydroxide structure types.

The $I 4 / m$ and $P 4 / m n c$ structures are shown in Fig. 3 and their structural details are given in Table 2 . Both comprise localised square planar hydrogen-bonded $(\mathrm{OH})_{4}$ units in a $\mathrm{Rb}^{+}$matrix; an alternative view is that $\mathrm{Rb}^{+}\left(\mathrm{OH}^{-}\right)$forms a distorted caesium chloride structure. Both structures have two $(\mathrm{OH})_{4}$ units per unit cell,

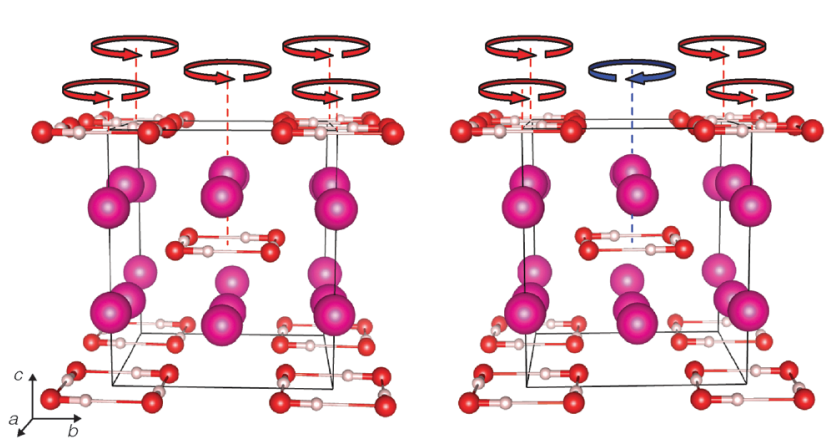

Fig. 3 Tetragonal high-pressure phases of $\mathrm{RbOH}-\mathrm{VI}$, shown at $P=10 \mathrm{GPa}$. $14 / \mathrm{m}(\mathrm{P} 4 / \mathrm{mnc})$ are shown on the left (right), and orientations of the hydrogen bond networks (see text) are indicated by coloured arrows.
Table 2 Optimized crystal structures of high-pressure $\mathrm{RbOH}$ and $\mathrm{CsOH}$ phases, all from ground state calculations

\begin{tabular}{|c|c|c|}
\hline Phase & Lattice constants $[\AA]$ & Atomic positions \\
\hline \multicolumn{3}{|c|}{ RbOH-IVb, $10 \mathrm{GPa}$} \\
\hline$\left(C m c 2_{1}\right)$ & $a=3.889, b=10.119, c=3.945$ & $\begin{array}{l}\mathrm{Rb}(0,0.141,0.279) \\
\mathrm{O}(0,0.408,0.292) \\
\mathrm{H}(0,0.476,0.110)\end{array}$ \\
\hline \multicolumn{3}{|c|}{$\mathrm{RbOH}-\mathrm{VI}, 10 \mathrm{GPa}$} \\
\hline$(I 4 / m)$ & $a=6.355, b=6.355, c=7.343$ & $\begin{array}{l}\mathrm{Rb}_{1}(0,0.5,0.25) \\
\mathrm{Rb}_{2}(0,0,-0.295) \\
\mathrm{O}(-0.237,-0.211,0) \\
\mathrm{H}(0.082,0.223,0)\end{array}$ \\
\hline$(P 4 / m n c)$ & $a=6.352, b=6.352, c=7.346$ & $\begin{array}{l}\mathrm{Rb}_{1}(0,0.5,0.25) \\
\mathrm{Rb}_{2}(0,0,-0.295) \\
\mathrm{O}(-0.234,0.214,0) \\
\mathrm{H}(-0.079,0.229,0)\end{array}$ \\
\hline \multicolumn{3}{|c|}{ CsOH-IVc, $10 \mathrm{GPa}$} \\
\hline$(P m c n)$ & $a=4.075, b=10.064, c=4.110$ & $\begin{array}{l}\mathrm{Cs}(0,0.144,0.251) \\
\mathrm{O}(0,0.416,0.232) \\
\mathrm{H}(0,0.478,0.416)\end{array}$ \\
\hline \multicolumn{3}{|c|}{ CsOH-VII, $10 \mathrm{GPa}$} \\
\hline$\left(P 2_{1} 2_{1} 2_{1}\right)$ & $a=4.079, b=5.694, c=7.662$ & $\begin{array}{l}\mathrm{Cs}(-0.2501,0.367,0.179) \\
\mathrm{O}(-0.237,-0.101,0.041) \\
\mathrm{H}(-0.081,0.211,-0.489)\end{array}$ \\
\hline$\left(P 2{ }_{1} \mathrm{Cn}\right)$ & $a=4.080, b=5.694, c=7.662$ & $\begin{array}{l}\mathrm{Cs}(-0.376,0.117,0.179) \\
\mathrm{O}(-0.392,-0.351,0.041) \\
\mathrm{H}(-0.209,-0.460,0.011)\end{array}$ \\
\hline
\end{tabular}

but they differ in the relative orientation of their hydroxyl groups: in $I 4 / \mathrm{m}$, all hydrogen bonds are oriented the same way (say, anticlockwise, as seen down the $c$ axis in Fig. 3), whereas, in $P 4 / m n c$, units in adjacent $a b$ planes are oriented in opposite senses (clockwise and anti-clockwise). In Fig. 3, arrows have been added to indicate schematically the sense of orientation of the hydrogen bonds. At room temperature and on a macroscopic scale RbOH-VI might well be proton-disordered (as was found in $\mathrm{KOH}-\mathrm{VI}$ ) - note the small enthalpy difference between $I 4 / m$ and $P 4 / m n c$ across the entire pressure range; the true high-pressure phase of RbOH-VI (at room temperature) could then be a more symmetric variant of both structures with partial proton occupancies, for instance with $I 4 / \mathrm{mmm}$ symmetry as found in KOH-VI. ${ }^{20}$

Without considering such disorder, finite-temperature stability of the new phases was investigated within the quasi-harmonic approximation, i.e. considering vibrational entropy contributions to the free energies of RbOH-IVb and the candidates for $\mathrm{RbOH}-\mathrm{VI}$ by evaluating

$$
F(T, V)=E_{0}(V)+k_{\mathrm{B}} T \int_{0}^{\infty} g(\omega) \ln \left[2 \sinh \left(\frac{\hbar \omega}{2 k_{\mathrm{B}} T}\right)\right] \mathrm{d} \omega .
$$

There, $g(\omega)$ is the phonon density of states obtained from supercell calculations. All phases presented here were found to be dynamically stable across their respective pressure range of stability, see Fig. S2 and S3 in the ESI $\dagger$ for phonon dispersions and densities of state. The resulting phase diagram is shown in Fig. 4. It incorporates experimental data on high-temperature phase transitions at $P=1 \mathrm{~atm}$ : from RbOH-IVb to proton-disordered $\mathrm{RbOH}-\mathrm{II}$ (monoclinic, $P 2_{1} / \mathrm{m}$ ) at $265 \mathrm{~K}$, to the cubic "free rotator" phase RbOH-I at $511 \mathrm{~K}$, and melting at $637 \mathrm{~K}$. The predicted highpressure $I 4 / \mathrm{m}$ phase should become stable around $P=10 \mathrm{GPa}$ 


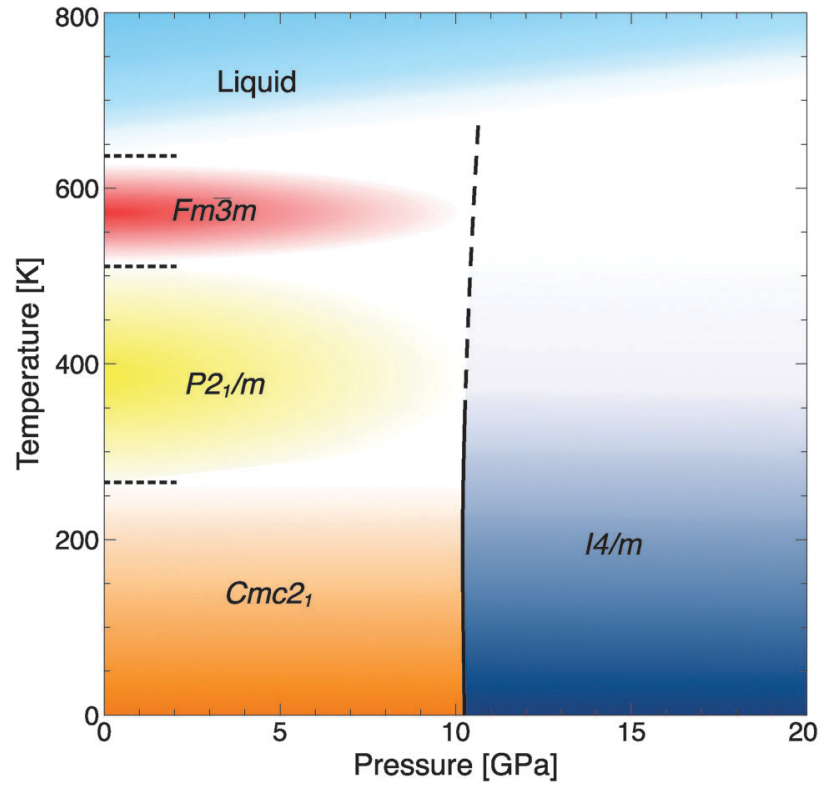

Fig. 4 Phase diagram of $\mathrm{RbOH}$, including the calculated transition line $\mathrm{RbOH}-\mathrm{IVb} \rightarrow \mathrm{VI}$ (solid-dashed line), experimental data at $P=1 \mathrm{~atm}$ (dotted lines), and estimates for the high-temperature phases' stability fields (coloured areas). ${ }^{4}$

over a range of temperatures. A transition to a proton-disordered high-temperature phase (like the RbOH-IVb $\rightarrow$ RbOH-II $\left(P 2_{1} / m\right)$ transition at $P=1 \mathrm{~atm}$ ) could increase the stability field of the high-pressure phase, but the entropy gain from two half-occupied proton sites would probably be similar to that of the low-pressure $P 2_{1} / m$ phase (this uncertainty is represented by drawing a dashed line in Fig. 4). The emergence of tetragonal $\mathrm{RbOH}-\mathrm{VI}$ also marks the deviation from layered structures in $\mathrm{RbOH}$ (not unexpected at high pressures), as $\mathrm{Rb}^{+}$and $\mathrm{OH}^{-}$form a distorted $\mathrm{CsCl}$ lattice in both candidate structures. This leads to a much more compact packing: at the transition pressure and in the ground state, $\mathrm{RbOH}-\mathrm{VI}$ is denser than $\mathrm{RbOH}-\mathrm{IVb}$ by $\Delta V / V=4.5 \%$. The emergence of $\mathrm{RbOH}-\mathrm{VI}$ also suggests a different quasi-cubic "free rotator" phase below the melting line at high pressures, which would be based on the B2-CsCl structure type (space group $\operatorname{Im} \overline{3} m$ ) instead of the B1-NaCl structure type (space group $F m \overline{3} m$ ).

The pressure where $\mathrm{RbOH}-\mathrm{VI}$ is stabilized in the ground state, about $10 \mathrm{GPa}$, compares reasonably well to the experimental result of $6.3 \mathrm{GPa}$, and calculations show a strong enthalpic driving force favoring the phase transition. This result is qualitatively independent of the exchange-correlation functional approximation used: using the local density approximation (LDA), ${ }^{34}$ the $I 4 / m$ phase becomes stable at $7.8 \mathrm{GPa}$, and using the optPBE-vdW functional (which includes dispersion corrections), ${ }^{35-38}$ the $I 4 / m$ phase is stabilized at $6.0 \mathrm{GPa}$, both with respect to the $\mathrm{RbOH}-\mathrm{IVb}$ phase. However, the two structures found to be suitable candidates are quite different from those proposed in the experimental studies. Note that the diffraction experiments used energy-dispersive X-rays, which make structural refinements quite difficult; and spectroscopic data has not been compared to candidate phases. The lattice parameters obtained in EDX experiments at 8.9 GPa are $a=3.206, b=6.371$, and $c=7.271 \AA^{15}{ }^{15}$ The orthorhombic deviation
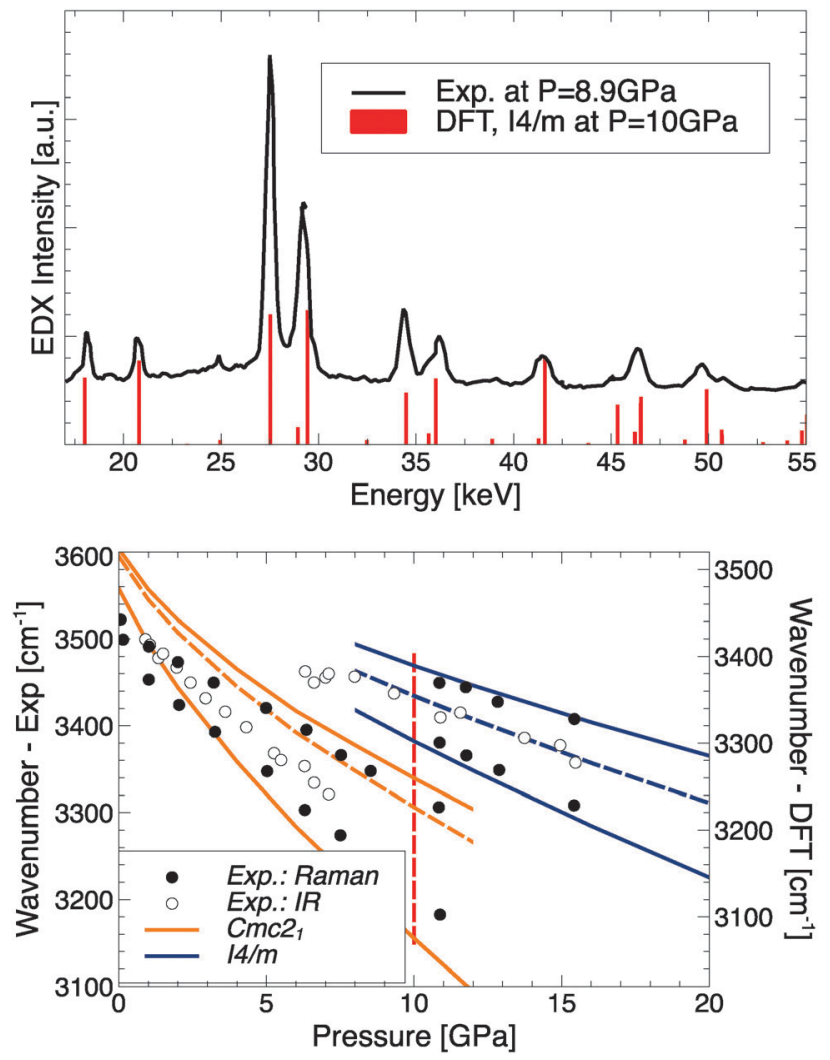

Fig. 5 Top: Comparing experimental EDX data from ref. 15 to simulated $\mathrm{X}$-ray intensities of $\mathrm{RbOH}-14 / \mathrm{m}$. Bottom: Comparing experimental IR/Raman peak positions from ref. 14 to calculated modes for $\mathrm{RbOH}-\mathrm{IVb}$ and $\mathrm{RbOH}-14 / \mathrm{m}$; solid (dashed) lines indicate Raman (IR) active modes, and the vertical red dashed line indicates the calculated transition pressure. Note calculated frequencies have been offset by $80 \mathrm{~cm}^{-1}$ to account for anharmonicity of the $\mathrm{OH}$ stretch mode. ${ }^{33}$

from a tetragonal structure with unit cell $(2 a, b, c)$ is very small, $2 a / b=1.006$, and arguably below the resolution of the EDX experiment. It is hence conceivable that $\mathrm{RbOH}-\mathrm{VI}$ is tetragonal (the calculated base lattice constant of the $I 4 / \mathrm{m}$ phase at $10 \mathrm{GPa}$ is $6.355 \AA$ ), and the top panel of Fig. 5 compares simulated peak positions and intensities of $\mathrm{RbOH}-\mathrm{VI}$ in the $I 4 / \mathrm{m}$ crystal structure to the EDX data - which give excellent agreement across the entire energy range, except for a small peak at $25 \mathrm{keV}$ (that the experimental structure assignment also could not fully explain).

In the bottom panel of Fig. 5, the experimental OH stretch frequencies as function of pressure are compared to calculated Raman- and IR-active modes for RbOH-IVb and the proposed $\mathrm{RbOH}-\mathrm{VI}$ structure. One finds very good agreement for the stretch modes in RbOH-IVb (after an ad hoc shift of $80 \mathrm{~cm}^{-1}$ to account for anharmonic effects not included in the calculations) ${ }^{33}$ and their decrease with pressure, and an equally good agreement for $\mathrm{RbOH}-\mathrm{VI}$, which exhibits a significant blueshift of all stretch modes at the phase transition. This suggests that the hydrogen bonds in $\mathrm{RbOH}-\mathrm{VI}$ are weaker than in RbOH-IVb, which is in agreement with structural arguments: at $P=10 \mathrm{GPa}$, the $\mathrm{O}-\mathrm{H} \cdots \mathrm{O}$ hydrogen bond lengths in RbOH-IVb are much shorter (1.73 $\AA$ ) than in RbOH-VI (1.86 $\mathrm{A})$, even though the latter is overall a much more compact structure. 


\subsection{Isosymmetric phase transition in $\mathrm{CsOH}$}

While there is no known high-pressure phase transition in $\mathrm{CsOH}$, the layered arrangement of $\mathrm{CsOH}-\mathrm{IVc}$ suggests that a more compact, three-dimensional network structure might become more stable under compression. A crystal structure search at $P=20 \mathrm{GPa}$ provides energetic arguments for a potential isosymmetric phase transition of $\mathrm{CsOH}$ around $10 \mathrm{GPa}$, to a phase one might call CsOH-VII. Fig. 6 shows the relative formation enthalpies of relevant phases. At low pressures, the anti- and ferroelectric structure types of $\mathrm{CsOH}-\mathrm{IVc}$ and $\mathrm{RbOH}-\mathrm{IVb}$ are quasi-degenerate as the most stable ground state structures for CsOH. Above $2 \mathrm{GPa}$, in the calculations, the $\mathrm{CsOH}-\mathrm{IVc}$ phase, which has $P 2_{1} 2_{1} 2_{1}$ symmetry at atmospheric pressure, optimizes to the higher Pmon symmetry. One potentially competitive high-pressure phase is a monoclinic $P 2_{1} / a$ structure that was suggested for $\mathrm{NaOH}-\mathrm{V},{ }^{13}$ but at $10 \mathrm{GPa}$ two other structures become more stable than $\mathrm{CsOH}-\mathrm{IVc}$. Note the energy scale in Fig. 6 is smaller by a factor of 20 than the same data shown for $\mathrm{RbOH}$ in Fig. 2. There is only a slight enthalpic driving force towards the formation of $\mathrm{CsOH}-\mathrm{VII}$, which is due to the relatively subtle structural changes compared to $\mathrm{CsOH}-\mathrm{IVc}$. However, the result is qualitatively unaffected by the choice of DFT approximation: using the LDA and optPBE-vdw exchange-correlation functionals, the same high-pressure phase transition is found to occur, respectively, at 4.6 and 6.6 GPa in the ground state; this is reasonable, given that the chemical bonding in $\mathrm{CsOH}-\mathrm{IVc}$ and $\mathrm{CsOH}-\mathrm{VII}$ is very similar (see below).

Again, the quasi-harmonic approximation gives an indication of the stability of the newly predicted phase at finite temperatures. The phase diagram, as shown in Fig. 7, reveals that the transition to the high-pressure phase should occur around 7 GPa at room temperature. Experimental information ${ }^{5}$ on the transition temperatures from $\mathrm{CsOH}-\mathrm{IVc}$ to proton-disordered $\mathrm{CsOH}-\mathrm{III}$ at $200 \mathrm{~K}$ (space group $\mathrm{Cmcm}$ ), to the "free rotator" phase $\mathrm{CsOH}-\mathrm{I}$ at $498 \mathrm{~K}$, and eventual melting at $612 \mathrm{~K}$ is included in the figure. Note that the underlying calculations only consider the transition $\mathrm{CsOH}-\mathrm{IVc} \rightarrow \mathrm{VII}$; in particular, possible stabilisation of a

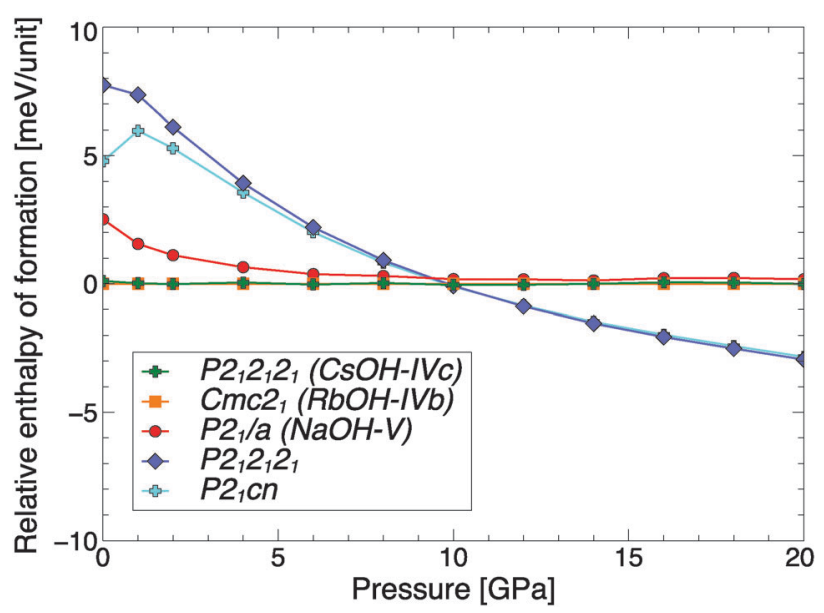

Fig. 6 Relative ground state enthalpies of formation per $\mathrm{CsOH}$ formula unit, as function of pressure.

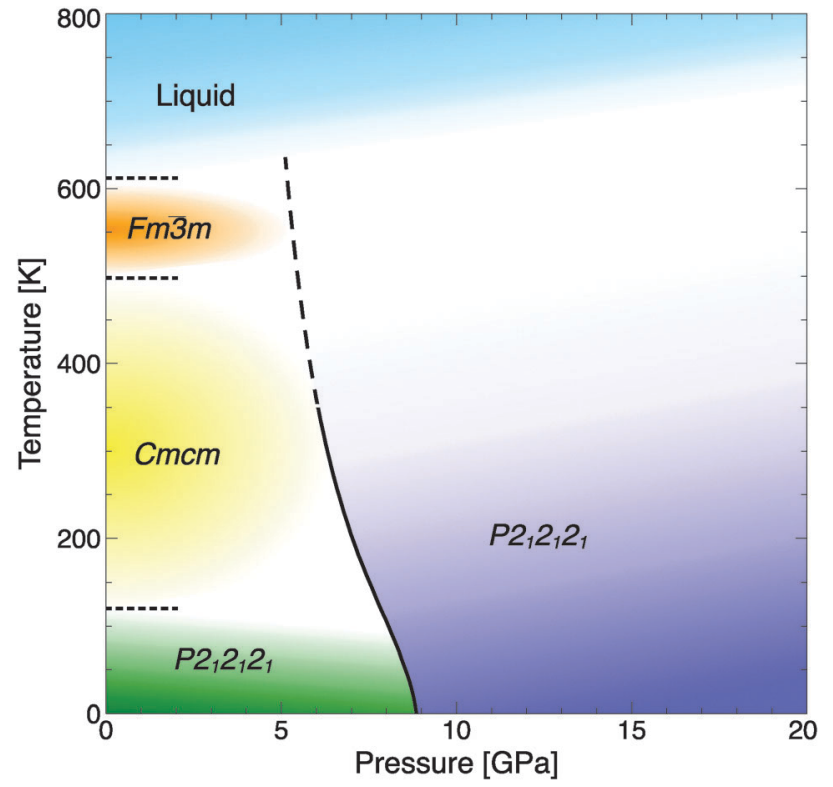

Fig. 7 Phase diagram of $\mathrm{CsOH}$, including the calculated transition line $\mathrm{CsOH}-\mathrm{IVc} \rightarrow \mathrm{VII}$ (solid-dashed line), experimental data at $P=1 \mathrm{~atm}$ (dotted lines), and estimates for the high-temperature phases' stability fields (coloured areas). ${ }^{5}$

proton-disordered phase $\mathrm{CsOH}-\mathrm{VII}^{\prime}$ at or around room temperature, and its stability relative to $\mathrm{CsOH}-\mathrm{III}$, are not included; the phase transition line is drawn as a dashed line to emphasize this uncertainty.

The most stable candidate for CsOH-VII above $10 \mathrm{GPa}$ in the ground state is orthorhombic, of $P 2_{1} 2_{1} 2_{1}$ symmetry, and is shown in Fig. 8. It features kinked hydrogen-bonded $\mathrm{OH}$ chains in a cation environment very similar to $\mathrm{CsOH}-\mathrm{IVc}$ (see Fig. 1(b)), but the cation 'channels' are laid out in a herringbone arrangement in the $b c$ plane as opposed to a parallel stacking in CsOH-IVc. This allows for a marginally more compact overall structure: at $10 \mathrm{GPa}$, the $P 2{ }_{1} 2_{1} 2_{1}$ structure is $0.2 \%$ denser per formula unit than CsOH-IVc. If one focuses on the cation coordination and arrangement, the $P 2_{1} 2_{1} 2_{1}$ structure clearly marks the deviation from layered structures, see Fig. 8(b), as edge-sharing $\mathrm{CsO}_{5}$ polyhedra form a three-dimensional network structure. The $\mathrm{OH}$ chains in CsOH-VII- $P 2_{1} 2_{1} 2_{1}$ have antiferroelectric order, and a
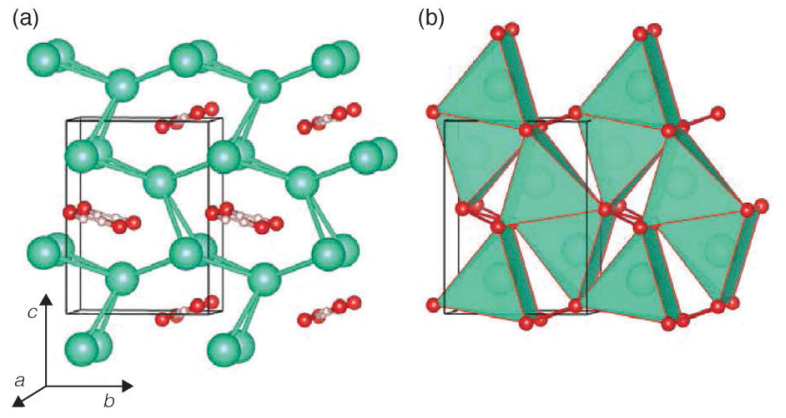

Fig. 8 Orthorhombic high-pressure phase of $\mathrm{CsOH}-\mathrm{VII}-P 2_{1} 2_{1} 2_{1}$, shown at $P=10 \mathrm{GPa}$. (a) Focus on hydrogen bonds and cation network; (b) focus on $\mathrm{CsO}_{5}$ polyhedral arrangement. 
ferroelectric equivalent of space group $P 2_{1} c n$ is quasi-degenerate with the $P 2_{1} 2_{1} 2_{1}$ phase at the relevant pressure range above $10 \mathrm{GPa}$, see Fig. 6 . The structural details of both phases are given in Table 2 . It is conceivable that $\mathrm{CsOH}-\mathrm{VII}$ will be proton-disordered at room temperature. Its $P 2_{1} 2_{1} 2_{1}$ structure has been found in calculations before, as a metastable high-pressure phase in $\mathrm{LiOH}^{21}$

A potential transition from $\mathrm{CsOH}-\mathrm{IVc}$ to $\mathrm{CsOH}-\mathrm{VII}$ should be very subtle in terms of equation of state or changes in spectroscopic signatures. Fig. 9 compares the two phases' volume per formula unit: $\mathrm{CsOH}-\mathrm{VII}-\mathrm{P} 2{ }_{1} 2_{1} 2_{1}$ is always more compact than $\mathrm{CsOH}-\mathrm{IVc}$, but only marginally so, with both phases approaching very similar molar volumes at high pressures. The equation of state of either phase agrees well with the experimental results by Otto and Holzapfel. ${ }^{15}$ Fig. 9 also shows calculated $\mathrm{OH}$ stretch modes as function of pressure, which suggest only minute differences between the two phases; this can be rationalized, because the $\mathrm{OH}$ group environments (with kinked one-dimensional hydrogen-bonded chains) are essentially identical. Both facts could explain why a pressureinduced phase transition was not seen in early experiments. ${ }^{14,15}$

It should be more straightforward to verify the presence of a transition in diffraction experiments. Fig. 10 compares simulated EDX peak positions and intensities of $\mathrm{CsOH}-\mathrm{IVc}$ and $\mathrm{CsOH}-\mathrm{P}_{1} 2_{1} 2_{1}$ with the experimental data by Otto and Holzapfel, as well as the evolution of the lattice constants with pressure. One finds that the optimized structure of $\mathrm{CsOH}-\mathrm{IVc}$ agrees very well with both sets of
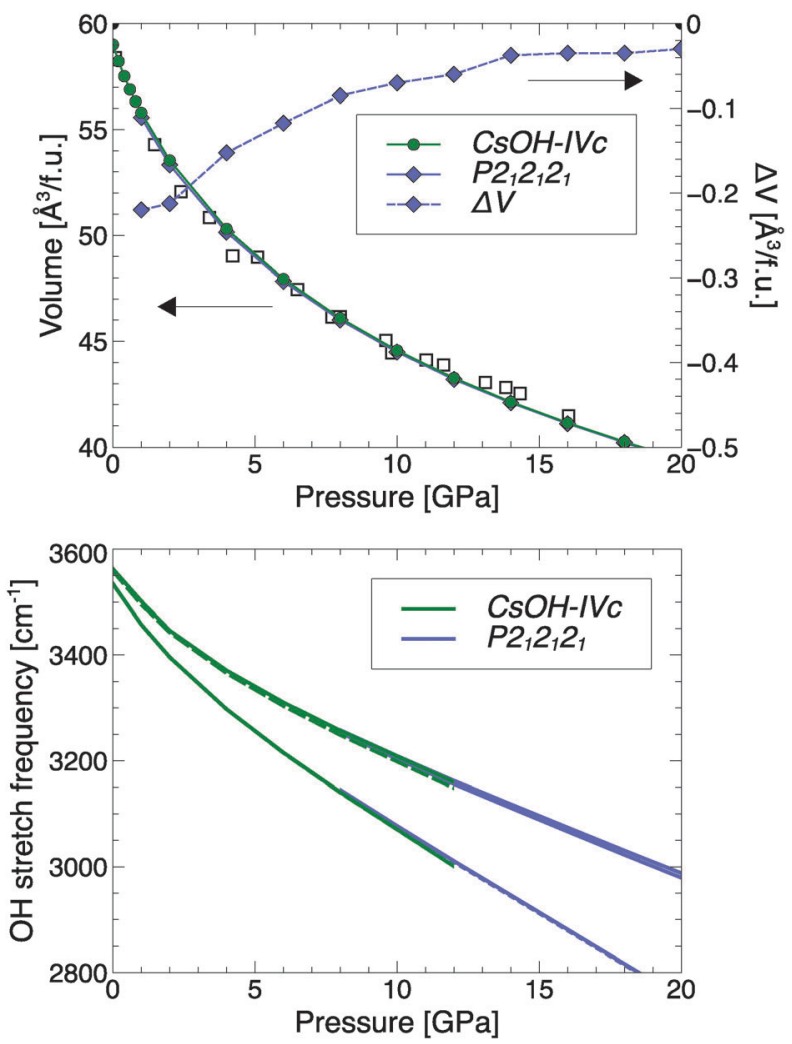

Fig. 9 Top: Calculated molar volumes of $\mathrm{CsOH}-\mathrm{IVc}$ and $\mathrm{CsOH}-\mathrm{VII}$, as well as their difference $\Delta V=V(\mathrm{CsOH}-\mathrm{VII})-V(\mathrm{CsOH}-\mathrm{IVc})$. Open symbols denote experimental values. ${ }^{15}$ Bottom: Calculated $\mathrm{OH}$ stretch modes in both phases, as function of pressure. Solid (dashed) lines are Raman + IR (IR only) active.
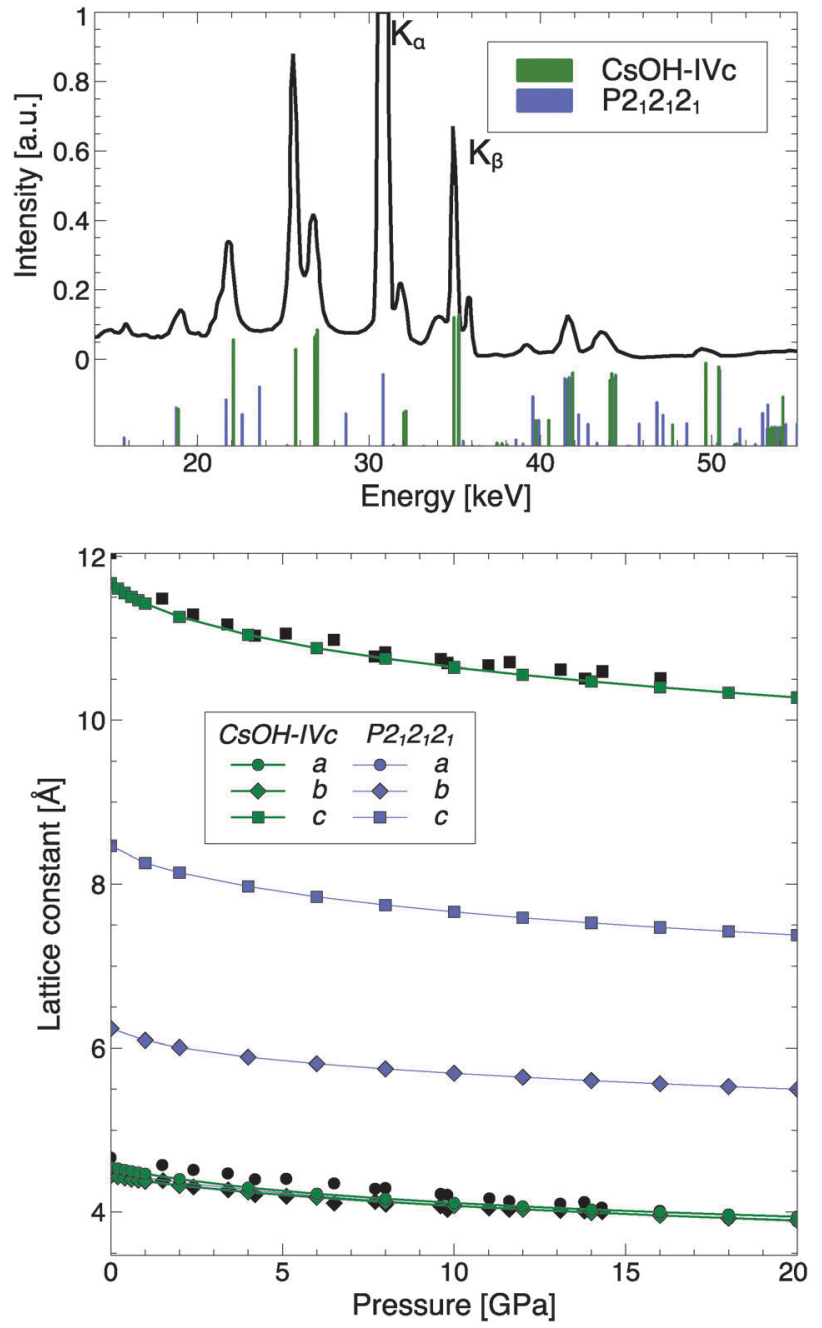

Fig. 10 Top: Experimental EDX spectrum at 9.6 GPa, and simulated EDX peaks at $10 \mathrm{GPa}$, for both $\mathrm{CsOH}-\mathrm{IVc}$ and $\mathrm{CsOH}-\mathrm{VII}$. Bottom: Calculated pressure evolution of lattice constants of both phases. Black symbols are experimental data points. ${ }^{15}$

experimental data. As the top panel of Fig. 10 shows, the predicted $P 2_{1} 2_{1} 2_{1}$ phase has a diffraction pattern very distinct from $\mathrm{CsOH}-\mathrm{IVc}$, and its presence in a sample chamber should be noticeable. The experimental pattern shows no sign of additional peaks that would indicate presence of the $P 2_{1} 2_{1} 2_{1}$ phase, and therefore suggests the stability of $\mathrm{CsOH}-\mathrm{IVc}$ (or, to be precise, CsOH-III) around $10 \mathrm{GPa}$ - while the present calculations predict a phase transition to $\mathrm{CsOH}-\mathrm{VII}$ (or -VII', with proton disorder) to occur under the same conditions. However, Otto and Holzapfel noted a broadening of several EDX lines above $10 \mathrm{GPa}$ that might indicate an approach of a structural phase transition. The transition could be unusually sluggish due to the small enthalpic driving force. Higher pressures were only investigated using spectroscopy, which (see above) is unlikely to provide clear evidence of a phase transition.

\section{Conclusions}

In summary, electronic structure calculations combined with crystal structure prediction methodology provide strong hints 
at hitherto unknown high-pressure phases in both $\mathrm{RbOH}$ and $\mathrm{CsOH}$ alkali hydroxides. For $\mathrm{RbOH}$, it is suggested that a previously identified high-pressure phase, $\mathrm{RbOH}-\mathrm{VI}$, has a completely different structure (which includes the hydrogen bond network) than assumed so far. Instead of being related to $\mathrm{NaOH}-\mathrm{V}$, evidence from energetics, simulated EDX patterns and $\mathrm{OH}$ vibrons suggests that $\mathrm{RbOH}-\mathrm{VI}$ has the same structure as $\mathrm{KOH}-\mathrm{VI}$, which was recently solved and features localised square-planar $(\mathrm{OH})_{4}$ units in an overall $\mathrm{B} 2$-like $\mathrm{Rb}^{+}(\mathrm{OH})^{-}$arrangement. The structural features of this phase could indicate a different high-temperature "free rotator" phase and even suggest a liquid-liquid phase transition in hot, compressed RbOH.

For $\mathrm{CsOH}$, a new high-pressure phase, $\mathrm{CsOH}-\mathrm{VII}$, is predicted to be stabilised around $10 \mathrm{GPa}$. This phase features a more subtle rearrangement of structural features than $\mathrm{RbOH}-\mathrm{VI}$, and hence a much smaller enthalpic driving force towards formation under high-pressure conditions. CsOH-VII marks, like RbOH-VI, the departure from layered structural motifs that are so dominant in all alkali hydroxides at atmospheric pressure. While there is no direct experimental evidence for CsOH-VII yet, the phase transition could be sluggish due to the small enthalpy gain upon its formation. Estimates of the finite-temperature phase diagram of either compound show that the proposed new high-pressure phases should be accessible at room temperature, albeit likely in proton-disordered form.

The pressure range of formation for both $\mathrm{RbOH}-\mathrm{VI}$ and $\mathrm{CsOH}-\mathrm{VII}$ is accessible by neutron powder diffraction, which should be the best experimental approach to conclusively solve those high-pressure phases. Their formation can probably also be verified using angle-dispersive X-ray diffraction of sufficient quality to enable a refinement. $\mathrm{CsOH}-\mathrm{VII}$ in particular exhibits a diffraction pattern quite different from $\mathrm{CsOH}-\mathrm{IVc}$.

The results presented here yet again extend the range of hydrogen bond networks possible in the alkali hydroxides under suitable conditions. The CsOH-VII phase was seen previously as a metastable candidate for LiOH-III, and with its emergence as a stable phase under compression one now finds hydroxyl hydrogen bond networks of dimensionality zero ( $\mathrm{KOH}-\mathrm{VI}$ and $\mathrm{RbOH}-\mathrm{VI}$, with localised $(\mathrm{OH})_{4}$ units), one (LiOH-III and CsOH-VII, with linear and kinked $(\mathrm{OH})_{\infty}$ chains), and two (LiOH-IV and $\mathrm{NaOH}-\mathrm{V}$, with bifurcated hydrogen bonds in $\mathrm{OH}$ layers), in a sequence of compounds that arguably do not differ by anything other than the cation size. This flexibility in hydrogen bond topologies could conceivably emerge in other systems as well, such as hydrous minerals, and could significantly influence their ionic conductivity and elasticity. It would be fascinating to study whether similar transitions occur, for instance, in the alkaline earth hydroxides brucite and portlandite, $\mathrm{Mg}(\mathrm{OH})_{2}$ and $\mathrm{Ca}(\mathrm{OH})_{2}$, which also form as layered compounds at atmospheric and low pressures.

\section{Acknowledgements}

The author wants to thank John S. Loveday and Roald Hoffmann for fruitful discussions. Computational resources provided by the UK National Supercomputing Service through the UKCP consortium (funded by EPSRC Grant EP/K01465X) and project ID d56, by the Condensed Matter Centre for Doctoral Training (funded by EPSRC Grant EP/L015110/1), and by the Royal Society (Research Grant RG150247) are gratefully acknowledged. Supplementary research data, in compliance with EPSRC research data policy, can be accessed at http://dx.doi.org/10.7488/ds/1413.

\section{References}

1 J. M. Kiat, G. Boemare, B. Rieu and D. Aymes, Solid State Commun., 1998, 108, 241-245.

2 H.-J. Bleif and H. Dachs, Acta Crystallogr., Sect. A: Cryst. Phys., Diffr., Theor. Gen. Crystallogr., 1982, 38, 470-476.

3 B. Mach, H. Jacobs and W. Schäfer, Z. Anorg. Allg. Chem., 1987, 553, 187-195.

4 H. Jacobs, B. Mach, H.-D. Lutz and J. Henning, Z. Anorg. Allg. Chem., 1987, 544, 28-54.

5 H. Jacobs, B. Mach, B. Harbrecht, H.-D. Lutz and J. Henning, Z. Anorg. Allg. Chem., 1987, 544, 55-73.

6 H. Dachs, Z. Kristall., 1959, 112, 60-67.

7 J. A. Ibers, J. Kumamoto and R. G. Snyder, J. Chem. Phys., 1960, 33, 1164.

8 T. J. Bastow, M. M. Elcombe and C. J. Howard, Solid State Commun., 1986, 57, 339-341.

9 T. J. Bastow, M. M. Elcombe and C. J. Howard, Solid State Commun., 1986, 59, 257-259.

10 T. J. Bastow, M. M. Elcombe and C. J. Howard, Solid State Commun., 1987, 62, 149-151.

11 T. J. Bastow, S. L. Segel and K. R. Jeffrey, Solid State Commun., 1991, 78, 565-568.

12 T. J. Bastow, M. M. Elcombe and C. J. Howard, Ferroelectrics, 1988, 79, 269-271.

13 D. M. Adams, A. G. Christy and J. Haines, J. Phys. Chem., 1992, 96, 8173-8176.

14 M. P. Krobok and W. B. Holzapfel, J. Phys.: Condens. Matter, 1994, 6, 9789-9806.

15 J. W. Otto and W. B. Holzapfel, J. Phys.: Condens. Matter, 1995, 7, 5461-5476.

16 J. S. Loveday, W. G. Marshall, R. J. Nelmes, S. Klotz, G. Hamel and J. M. Besson, J. Phys.: Condens. Matter, 1996, 8, L597-L604.

17 R. J. Angel, D. J. Frost, N. L. Ross and R. Hemley, Phys. Earth Planet. Inter., 2001, 127, 181-196.

18 Q. Williams and R. J. Hemley, Annu. Rev. Earth Planet. Sci., 2001, 29, 365-418.

19 R. A. Eggleton, J. N. Boland and A. E. Ringwood, Geochem. J., 1978, 12, 191-194.

20 A. Hermann, M. Guthrie, R. J. Nelmes and J. S. Loveday, J. Chem. Phys., 2015, 143, 244706.

21 A. Hermann, N. W. Ashcroft and R. Hoffmann, J. Chem. Phys., 2014, 141, 024505.

22 D. C. Lonie and E. Zurek, Comput. Phys. Commun., 2011, 182, 372-387.

23 G. Kresse and J. Furthmüller, Phys. Rev. B: Condens. Matter Mater. Phys., 1996, 54, 11169-11186. 
24 E. Zurek, R. Hoffmann, N. W. Ashcroft, A. R. Oganov and A. O. Lyakhov, Proc. Natl. Acad. Sci. U. S. A., 2009, 106, 17640-17643.

25 A. Hermann, N. W. Ashcroft and R. Hoffmann, Proc. Natl. Acad. Sci. U. S. A., 2012, 109, 745-750.

26 A. Hermann, A. Suarez-Alcubilla, I. G. Gurtubay, L.-M. Yang, A. Bergara, N. W. Ashcroft and R. Hoffmann, Phys. Rev. B: Condens. Matter Mater. Phys., 2012, 86, 144110.

27 A. Hermann, A. McSorley, N. W. Ashcroft and R. Hoffmann, J. Am. Chem. Soc., 2012, 134, 18606.

28 A. Hermann and P. Schwerdtfeger, J. Phys. Chem. Lett., 2014, 5, 4336-4342.

29 P. E. Blöchl, Phys. Rev. B: Condens. Matter Mater. Phys., 1994, 50, 17953-17979.

30 G. Kresse and D. Joubert, Phys. Rev. B: Condens. Matter Mater. Phys., 1999, 59, 1758-1775.

31 D. Alfè, Comput. Phys. Commun., 2009, 180, 2622-2633.

32 P. Giannozzi, S. Baroni, N. Bonini, M. Calandra, R. Car, C. Cavazzoni, D. Ceresoli, G. L. Chiarotti, M. Cococcioni, I. Dabo, A. Dal Corso, S. de Gironcoli, S. Fabris, G. Fratesi, R. Gebauer, U. Gerstmann, C. Gougoussis, A. Kokalj, M. Lazzeri,
L. Martin-samos, N. Marzari, F. Mauri, R. Mazzarello, S. Paolini, A. Pasquarello, L. Paulatto, C. Sbraccia, S. Scandolo, G. Sclauzero, A. P. Seitsonen, A. Smogunov, P. Umari, R. M. Wentzcovitch, A. D. Corso, S. D. Gironcoli, S. Fabris, G. Fratesi, R. Gebauer, U. Gerstmann, C. Gougoussis, A. Kokalj, M. Lazzeri, L. Martin-samos, N. Marzari, F. Mauri, R. Mazzarello, S. Paolini, A. Pasquarello, L. Paulatto, C. Sbraccia, A. Smogunov, P. Umari and R. M. Wentzcovitch, J. Phys.: Condens. Matter, 2009, 21, 395502.

33 K. Hermansson, G. Gajewski and P. D. Mitev, J. Phys. Chem. A, 2008, 112, 13487-13494.

34 J. P. Perdew and A. Zunger, Phys. Rev. B: Condens. Matter Mater. Phys., 1981, 23, 5048-5079.

35 M. Dion, H. Rydberg, E. Schröder, D. C. Langreth and B. I. Lundqvist, Phys. Rev. Lett., 2004, 92, 246401.

36 G. Román-Pérez and J. M. Soler, Phys. Rev. Lett., 2009, 103, 096102.

37 J. Klimeš, D. R. Bowler and A. Michaelides, J. Phys.: Condens. Matter, 2010, 22, 022201.

38 J. Klimeš, D. Bowler and A. Michaelides, Phys. Rev. B: Condens. Matter Mater. Phys., 2011, 83, 195131. 